\title{
A PROBLEM IN ELECTRICAL PROSPECTION AND A $n$-DIMENSIONAL BORG-LEVINSON THEOREM
}

\author{
SAGUN CHANILLO \\ (Communicated by Barbara L. Keyfitz) \\ Dedicated to M. Cotlar on his 75 th birthday
}

\begin{abstract}
We show that the Dirichlet to Neumann map for $-\Delta u+v u=0$, determines the potential $v(x)$, for $v(x)$ satisfying the condition of $\mathrm{C}$. Fefferman and D. Phong.
\end{abstract}

We shall consider here a bounded domain $\Omega \subset \mathbf{R}^{n}, n \geq 3$ with smooth boundary. Consider then the equation in $\Omega$ given by $-\Delta u+v u=0$. Define the Dirichlet to Neumann map $\Lambda_{v}$ on $\partial \Omega$ given by

$$
\Lambda_{v}(f)=\frac{\partial u}{\partial \nu}, \frac{\partial u}{\partial \nu} \text { is the outward normal derivative }
$$

and $u$ solves the Dirichlet problem $-\Delta u+u v=0$ in $\Omega$ and $\left.u\right|_{\partial \Omega}=f$.

We recall, ([F], [CW]) the definition of the C. Fefferman, D. Phong class. We say $v \in F_{p}$ if for all cubes $Q \subset \mathbf{R}^{n}$,

$$
\|v\|_{F_{p}}=\sup _{Q}|Q|^{2 / n}\left[\frac{1}{|Q|} \int_{Q}|v|^{p}\right]^{1 / p}<\infty .
$$

We remark that $L^{n / 2}\left(\mathbf{R}^{n}\right) \subset F_{p}$ for $p \leq n / 2$, and likewise $L^{n / 2, \infty} \subset F_{p}$, $p<n / 2$. The containments are strict as $v=f(x /|x|)|x|^{-2}, f \in L^{p}\left(S^{n-1}\right)$, $p>(n-1) / 2$ is not in $L^{n / 2, \infty}$ but $v \in F_{p}, p>(n-1) / 2$. The main result proved here is as follows.

Theorem. Suppose $\left\|v_{i}\right\|_{F_{p}} \leq \varepsilon(n), p>(n-1) / 2, i=1,2$. Assume that $\Lambda_{v_{1}}=\Lambda_{v_{2}}$, then $v_{1}=v_{2}$ in $\Omega$.

Remark. In the theorem above it is enough to assume that $v_{i}$ are supported in $\Omega$.

The one-dimensional result is due to [B], [L]. If $v_{i} \in L^{\infty}$ the result is due to [NSU] and [HN]. The two-dimensional result is in $\left[\mathrm{SU}_{1}\right]$ and the $C^{\infty}$ case in

Received by the editors February 8, 1989 and, in revised form, May 9, 1989.

1980 Mathematics Subject Classification (1985 Revision). Primary 35P10, 42B20, 35B60, 35J10; Secondary 35J05, 35P20.

Supported in part by NSF grant DMS- 8803493 . 


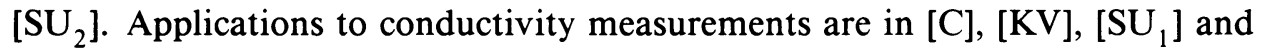
$\left[\mathrm{SU}_{2}\right] .[\mathrm{KV}]$ also treats the case where $v_{i}$ are analytic.

The smallness assumption on the $F_{p}$ norm is not needed if $v_{i} \in L^{p}(\Omega)$, $p>n / 2$. We wish to thank D. Jerison and C. Kenig for pointing this out to us and also include their proof of this observation after the end of the proof in the main theorem above.

We recall the following inequality from [CS].

Theorem 1. Let $f \in C_{0}^{\infty}\left(\mathbf{R}^{n}\right), v>0$ and $v \in F_{p}, p>(n-1) / 2$. For $z \in \mathbf{C}^{n}, \gamma \in \mathbf{C}$, define $Q(D)=\Delta+z \cdot \nabla+\gamma$. Then

$$
\int_{\mathbf{R}^{n}}|f|^{2} v \leq c \int_{\mathbf{R}^{n}}|Q(D) f|^{2} v^{-1}
$$

where $c$ is independent of $f, z, \gamma$.

We use the theorem stated above to prove the lemma that follows.

Lemma 1. Let $v_{i} \in F_{p} \cap L^{1}, p>(n-1) / 2$, and $\left\|v_{i}\right\|_{F_{p}} \leq \varepsilon, i=1,2$. Let $z \in \mathbf{C}^{n}$ with $z \cdot z=0$. Let $V(x)=\left|v_{1}\right|+\left|v_{2}\right|+\delta\left(1+|x|^{2}\right)^{-n}, \delta>0$ and small. Let $L_{V}^{2}=\left\{f: \int_{\mathbf{R}^{n}}|f|^{2} V<\infty\right\}$. Then,

(a) there is a unique solution to $-\Delta+v_{i}$ of the form,

$$
u_{i}(x)=e^{z \cdot x} m_{z, i}(x), i=1,2 \text { with } m_{z, i} \text { in the space } L_{V}^{2} .
$$

(b) $\int_{\mathbf{R}^{n}}\left|m_{z, i}\right|^{2} V \leq c$, uniformly in $z, i=1,2$.

(c) $m_{z, i}(x) \rightarrow 1, i=1,2$ weakly in $L_{V}^{2}$ as $\left|z_{k}\right| \rightarrow \infty$, for some sequence $z_{k}$.

Proof. Substituting $u=e^{z \cdot x} m_{z, i}(x)$ into $-\Delta u+v_{i} u=0$, we note that $m_{z, i}(x)$ satisfies the equation

$$
-\Delta m_{z, i}+(z \cdot \nabla) m_{z, i}+v_{i} m_{z, i}=0 .
$$

Therefore, $m_{z, i}$ satisfies the integral equation,

$$
m_{z, i}=1+G_{z}\left(v_{i} m_{z, i}\right), \quad i=1,2
$$

where $G_{z}$ denotes the Green function for $-\Delta+z \cdot \nabla$. Define $T_{i} f(x)=1+$ $G_{z}\left(v_{i} f\right)(x)$. It will be enough for us to show $T_{i}$ has a fixed point on the Banach space $L_{V}^{2}$, thus showing (a). In fact we show $T_{i}$ is a contraction on $L_{V}^{2}$ and thus the uniqueness assertion (a) of Lemma 1 also follows. Since $v_{1}, v_{2} \in L^{1}$, $V \in L^{1}$. Since $v_{1}, v_{2}, \delta\left(1+|x|^{2}\right)^{-n} \in F_{p}, p>(n-1) / 2$, we have $\|V\|_{F_{p}} \leq \varepsilon$, for $p>(n-1) / 2$. Moreover $V>0$. Thus, Theorem 1 is applicable with $v=V$. Moreover $\left|v_{i}\right| \leq V, i=1,2$. 
Thus,

$$
\begin{aligned}
\int_{\mathbf{R}^{n}}\left|T_{i} f\right|^{2} V & \leq \int_{\mathbf{R}^{n}} V+\int_{\mathbf{R}^{n}}\left|G_{z}\left(v_{i} f\right)\right|^{2} V \\
& \leq c+\varepsilon \int_{\mathbf{R}^{n}}\left|v_{i} f\right|^{2} V^{-1} \leq c+\varepsilon \int_{\mathbf{R}^{n}}|f|^{2} V^{2} V^{-1} \\
& \leq \int_{\mathbf{R}^{n}}|f|^{2} V<\infty .
\end{aligned}
$$

Similarly,

$$
\begin{aligned}
\int_{\mathbf{R}^{n}}\left|T_{i}(f-g)\right|^{2} V & \leq \int_{\mathbf{R}^{n}} \mid G_{z}\left(\left.v_{i}(f-g)\right|^{2} V \leq \varepsilon \int_{\mathbf{R}^{n}}|f-g|^{2}\left|v_{i}\right|^{2} V^{-1}\right. \\
& \leq \varepsilon \int_{\mathbf{R}^{n}}|f-g|^{2} V .
\end{aligned}
$$

Thus $T_{i}$ is a contraction and the existence and uniqueness of $m_{z, i}$ is assured. We now show (b). By (1),

$$
\begin{aligned}
\int_{\mathbf{R}^{n}}\left|m_{z, i}\right|^{2} V & \leq c \int_{\mathbf{R}^{n}} V+c \int_{\mathbf{R}^{n}}\left|G_{z}\left(v_{i} m_{z, i}\right)\right|^{2} V \\
& \leq c_{1} \int_{\mathbf{R}^{n}} V+\varepsilon c_{2} \int_{\mathbf{R}^{n}}\left|m_{z, i}\right|^{2} V
\end{aligned}
$$

where $c_{1}, c_{2}$ do not depend on $z$. Thus for small $\varepsilon$, and since from (a), $\int_{\mathbf{R}^{n}}\left|m_{z, i}\right|^{2} V<\infty$, we see,

$$
\int_{\mathbf{R}^{n}}\left|m_{z, i}\right|^{2} V \leq c, \quad \text { uniformly in } z .
$$

We now prove (c). Note the multiplier for $G_{z}$ given by $\left(|\xi|^{2}+i z \cdot \xi\right)^{-1} \rightarrow 0$ as $|z| \rightarrow \infty$. Next we note by (b),

$$
\int_{\mathbf{R}^{n}}\left|G_{z}\left(v_{i} m_{z, i}\right)\right|^{2} V \leq \varepsilon \int_{\mathbf{R}^{n}}\left|m_{z, i}\right|^{2} V \leq c .
$$

Thus there is a sequence $z_{k},\left|z_{k}\right| \rightarrow \infty$, so that $G_{z_{k}}\left(v_{i} m_{z_{k}, i}\right) \rightarrow 0$ weakly in $L_{V}^{2}$. Since (1) holds, it follows that $m_{z_{k}, i}-1$ as $\left|z_{k}\right| \rightarrow \infty$ in $L_{V}^{2}$. The lemma is now proved.

Lemma 2. Extend $v_{1}$ and $v_{2}$ to be zero outside $\Omega$. Let $u_{i}$ be the unique solutions of Lemma 1 to $-\Delta+v_{i}, i=1,2$. If $\Lambda_{v_{1}}=\Lambda_{v_{2}}$, then $u_{1}=u_{2}$ in $\mathbf{R}^{n} \backslash \Omega$.

Proof. Recall, [F], [CW], that if $\left\|v_{i}\right\|_{F_{p}} \leq \varepsilon, p>1$, then for $f \in C_{0}^{\infty}\left(\mathbf{R}^{n}\right)$,

$$
\int_{\mathbf{R}^{n}}|f|^{2}\left|v_{i}\right| \leq \varepsilon \int_{\mathbf{R}^{n}}|\nabla f|^{2}
$$

Thus the bilinear form,

$$
\int_{\Omega} \nabla u \cdot \nabla \varphi+\int_{\Omega} u \varphi v_{2}
$$


is coercive and continuous for $u, \varphi \in H_{0}^{1}(\Omega)$. As,

$$
\int_{\Omega}\left|m_{z, 1}\right|^{2}\left|v_{2}\right| \leq \int_{\mathbf{R}^{n}}\left|m_{z, 1}\right|^{2}\left|v_{2}\right| \leq \int_{\mathbf{R}^{n}}\left|m_{z, 1}\right|^{2} V<\infty,
$$

and $u_{1}=e^{z \cdot x} m_{z, 1}$, we get $\int_{\Omega}\left|u_{1}\right|^{2}\left|v_{2}\right|<\infty$. Thus, the Dirichlet problem,

$$
\begin{aligned}
-\Delta u+u v_{2} & =0 & & \text { in } \Omega \\
u & =u_{1} & & \text { on } \partial \Omega
\end{aligned}
$$

has a unique solution $u$, such that $\int_{\Omega}|u|^{2}\left|v_{2}\right|<\infty$.

Since $u-u_{1}$ has compact support, by (2), as $V \in F_{p}, p>1$,

$$
\int_{\Omega}\left|u-u_{1}\right|^{2} V \leq \int_{\Omega}\left|\nabla\left(u-u_{1}\right)\right|^{2}<\infty
$$

On $\Omega,\left|u_{1}\right| \leq c\left|m_{z, 1}\right|$, thus, $\int_{\Omega}|u|^{2} V<\infty$ by Lemma 1 .

Define,

$$
\Phi= \begin{cases}u & \text { in } \Omega \\ u_{1} & \text { in } \mathbf{R}^{n} \backslash \Omega .\end{cases}
$$

Since $\Lambda_{v_{2}}\left(u_{1}\right)=\partial u / \partial \nu=\Lambda_{v_{1}}\left(u_{1}\right)=\partial u_{1} / \partial \nu, \Phi$ is a solution to $\mathbf{R}^{n}$ to $-\Delta+v_{2}$. Writing $\Phi=e^{z \cdot x}\left[e^{-z \cdot x} \Phi\right]=e^{z \cdot x} M_{z}(x)$, we see that $M_{z}(x)=m_{z, 1}(x)$ in $\mathbf{R}^{n} \backslash \Omega$, and since $\int_{\Omega}|u|^{2} V<\infty$, it follows that $\int_{\mathbf{R}^{n}}\left|M_{z}\right|^{2} V<\infty$. By the uniqueness assertion of Lemma $1, M_{z}=m_{z, 2}$, and thus $\Phi=u_{2}$, in particular $u_{1}=u_{2}$ in $\mathbf{R}^{n} \backslash \Omega$.

We are now in a position to prove our main theorem.

Proof. Fix $l \in \mathbf{Z}^{n}$. Choose $k, e \in \mathbf{R}^{n}$, so that $|k|=|l-e|, k \cdot e=k \cdot l=e \cdot l=$ 0 . This choice forces $|k|=|l+e|$. Let $z=\frac{1}{2}(-k+i(l-e)), \tilde{z}=\frac{1}{2}(k+i(l+e))$. Note $z \cdot z=\tilde{z} \cdot \tilde{z}=0$. We shall use Green's theorem in the form,

$$
\int_{\Omega}(w \Delta f-f \Delta w)=\int_{\partial \Omega}\left[w \frac{\partial f}{\partial \nu}-f \frac{\partial w}{\partial \nu}\right] d \sigma
$$

with the choice $w=e^{\bar{z} \cdot x}, f=u_{i}=e^{z \cdot x} m_{z, i}$. Let $D_{\rho}$ be a collar neighborhood of $\partial \Omega$ with thickness $\rho$. Let $\partial D_{\rho} \cap \Omega=\partial D_{\rho, 1}$ and $\partial D_{\rho} \cap\left(\mathbf{R}^{n} \backslash \Omega\right)=\partial D_{\rho, 2}$. Note

$$
\int_{\Omega} e^{\dot{z} \cdot x} v_{i} u_{i}=-\int_{\Omega} e^{\dot{z} \cdot x} \Delta u_{i}=\int_{\partial \Omega} e^{\tilde{z} \cdot x}\left[(\tilde{z} \cdot \nu) u_{i}-\frac{\partial u_{i}}{\partial \nu}\right] d \sigma .
$$

We now show that,

$$
\int_{\partial \Omega} e^{\tilde{z} \cdot x}\left[(\tilde{z} \cdot \nu) u_{1}-\frac{\partial u_{1}}{\partial \nu}\right] d \sigma=\int_{\partial \Omega} e^{\tilde{z} \cdot x}\left[(\tilde{z} \cdot \nu) u_{2}-\frac{\partial u_{2}}{\partial \nu}\right] d \sigma .
$$

Temporarily assume (4). Combining (3) and (4) we see

$$
\int_{\Omega} e^{\dot{z} \cdot x} v_{1} u_{1}=\int_{\Omega} e^{\vdots \cdot x} v_{2} u_{2} \text {. }
$$


Since $u_{i}=e^{z \cdot x} m_{z, i}$, we get

$$
\int_{\Omega} e^{i l \cdot x} m_{z, 1} v_{1}=\int_{\Omega} e^{i l \cdot x} m_{z, 2} v_{2} .
$$

Letting $|z| \rightarrow \infty$ and using (c) of Lemma 1, we conclude

$$
\int_{\Omega} e^{i l \cdot x} v_{1}=\int_{\Omega} e^{i l \cdot x} v_{2} \text {. }
$$

This shows $v_{1}=v_{2}$. We now show (4). We apply Green's theorem to the collar neighborhood $D_{\rho}$. Since $v_{1}=v_{2}=0$ in $\mathbf{R}^{n} \backslash \Omega, u_{1}$ and $u_{2}$ are harmonic and $C^{\infty}$ in $\mathbf{R}^{n} \backslash \Omega$. By Lemma $2, u_{1}=u_{2}$ in $\mathbf{R}^{n} \backslash \Omega$. Thus

$$
\int_{\partial D_{\rho, 2}} e^{\tilde{z} \cdot x}\left[(\tilde{z} \cdot \nu) u_{1}-\frac{\partial u_{1}}{\partial \nu}\right] d \sigma=\int_{\partial D_{\rho, 2}} e^{\tilde{z} \cdot x}\left[(\tilde{z} \cdot \nu) u_{2}-\frac{\partial u_{2}}{\partial \nu}\right] d \sigma .
$$

So by Green's theorem

$$
\int_{\partial D_{\rho, 1}} e^{\tilde{z} \cdot x}\left[(\tilde{z} \cdot \nu)\left(u_{1}-u_{2}\right)-\frac{\partial u_{1}}{\partial \nu}+\frac{\partial u_{2}}{\partial \nu}\right] d \sigma=\int_{D_{\rho}} e^{\tilde{z} \cdot x}\left(v_{1} u_{1}-v_{2} u_{2}\right) .
$$

But $\int_{D_{\rho}}\left|u_{i}\right|^{2} V<\infty$. Thus as $\rho \rightarrow 0$, the integral on the right side converges to zero. Thus the integral on the left side converges to zero. But in the limit the integral on the left side is exactly the difference of the two integrals in (4). This establishes (4).

We now give the argument by $D$. Jerison and C. Kenig. In essence we show that a form of Lemma 1 holds with no smallness assumption if $v_{i} \in L^{r}(\Omega)$, $r>n / 2$.

We begin with,

Lemma 3. Let $2 /(n+1) \leq(q-2) / q \leq 2 / n$. Let $|z|=1$, and $z \cdot z=0$. Then for $1 / p+1 / q=1$,

$$
\left\|G_{z} f\right\|_{L^{q}\left(\mathbf{R}^{n}\right)} \leq c\|f\|_{L^{p}\left(\mathbf{R}^{n}\right)}
$$

where $\widehat{G_{z}} f(\xi)=\left(|\xi|^{2}+z \cdot \xi\right)^{-1} \hat{f}(\xi)$ and $c$ is independent of $f$ and $z$.

Proof. We assume w.l.o.g. that

$$
\widehat{G}_{z} f(\xi)=\left(|\xi|^{2}-2 \xi_{1}+2 i \xi_{2}\right)^{-1} \hat{f}(\xi) \text {. }
$$

By changing variables in $\xi_{1}, \xi_{1} \rightarrow\left(\xi_{1}-1\right)$ we can assume that $\widehat{G_{z}} f(\xi)=$ $\left(|\xi|^{2}+1+2 i \xi_{2}\right)^{-1} \hat{f}(\xi)$. Since $1 / p+1 / q=1,(q-2) / q=1 / p-1 / q$, and thus under the hypothesis of the lemma, $2 /(n+1) \leq 1 / p-1 / q \leq 2 / n$. We may thus apply Theorem 2.4 in [KRS] to conclude Lemma 3.

From Lemma 3 we deduce the next lemma. The notation we adopt is identical to Lemma 1.

Lemma 4. Let $w_{z, i}(x)=m_{z, i}(x)-1$. Let $v_{i} \in L^{r}(\Omega), r>n / 2$, and $z \cdot z=0$. Let $2 /(n+1) \leq(q-2) / q=1 / r<2 / n$. Then, for $|z|$ large,

(a) there is a unique solution to $-\Delta+v_{i}$ of the form $u_{i}(x)=e^{z \cdot x} m_{z, i}(x)$, with $\left\|w_{z, i}\right\|_{L^{q}\left(\mathbf{R}^{n}\right)} \leq c$ uniformly in $z$.

(b) $\left\|w_{z, i}\right\|_{L^{q}\left(\mathbf{R}^{n}\right)} \rightarrow 0$ as $|z| \rightarrow \infty$. 
Proof. From (1) we readily see that $w_{z, i}$ satisfies

$$
w_{z, i}+G_{z}\left(v_{i} w_{z, i}\right)=G_{z}\left(v_{i} \chi_{\Omega}\right) .
$$

Let $M_{v_{i}}(f)=v_{i} f$, the multiplication by $v_{i}$ operator. The identity above can be rewritten as,

$$
\left(I+G_{z} M_{v_{i}}\right)\left(w_{z, i}\right)=G_{z} M_{v_{i}}\left(\chi_{\Omega}\right),
$$

where $I=$ identity operator. We now claim that for $\alpha=2-n / r>0$, and for $c$ independent of $z$,

$$
\left\|G_{z} M_{v_{i}}(f)\right\|_{L^{q}\left(\mathbf{R}^{n}\right)} \leq c|z|^{-\alpha}\|f\|_{L^{q}\left(\mathbf{R}^{n}\right)} .
$$

Temporarily assume (6) and note that for large $|z|, I+G_{z} M_{v_{i}}$ is invertible on $L^{q}\left(\mathbf{R}^{n}\right)$, and

$$
\left\|G_{z} M_{v_{i}}\left(\chi_{\Omega}\right)\right\|_{L^{q}\left(\mathbf{R}^{n}\right)} \leq c|z|^{-\alpha}|\Omega|^{1 / q},
$$

$c$ independent of $z$. Thus the uniqueness and existence of $w_{z, i}$ follows from (5) and $\left\|w_{z, i}\right\|_{L^{q}\left(\mathbf{R}^{n}\right)} \leq c|z|^{-\alpha}$. So we are reduced to checking (6). Let $\delta=|z|$, and $T_{\delta} f(x)=f(\delta x)$. We note $G_{z}=\delta^{-2} T_{\delta} G_{z \delta^{-1}} T_{\delta^{-1}}$ by a change of variables, and moreover $\left\|T_{\delta} f\right\|_{L^{s}\left(\mathbf{R}^{n}\right)}=\delta^{-n / s}\|f\|_{L^{s}\left(\mathbf{R}^{n}\right)}$. Thus,

$$
\left\|G_{z} M_{v_{i}}(f)\right\|_{L^{q}}=\delta^{-2-n / q}\left\|G_{z \delta^{-1}} T_{\delta^{-1}} M_{v_{i}} f\right\|_{L^{q}} .
$$

By Lemma 3, the right side above is at most

$$
c \delta^{-2-n / q}\left\|T_{\delta^{-1}} M_{v_{i}}(f)\right\|_{L^{p}} \leq c \delta^{-2-n / q+n / p}\left\|v_{i} f\right\|_{L^{p}}, \quad 1 / p+1 / q=1 .
$$

Now $r^{-1}=1-2 q^{-1}=p^{-1}-q^{-1}$, because $p^{-1}+q^{-1}=1$. So the right side above is at most $c \delta^{-2+n / r}\left\|v_{i} f\right\|_{L^{p}}$. Now applying Holder's inequality with exponents $r / p$ and $r /(r-p)=q / p$,

$$
\begin{aligned}
c \delta^{-2+n / r}\left\|v_{i} f\right\|_{L^{p}} & \leq c \delta^{-2+n / r}\left\|v_{i}\right\|_{L^{r}(\Omega)}\|f\|_{L^{q}\left(\mathbf{R}^{n}\right)} \\
& \leq|z|^{-\alpha}\|f\|_{L^{q}\left(\mathbf{R}^{n}\right)} .
\end{aligned}
$$

Thus we have (6), and Lemma 4 follows.

Using Lemma 4 we may conclude the fact that $\Lambda_{v}$ determines $v$ exactly as before.

\section{REFERENCES}

[BC] R. Beals and R. Coifman, Multidimensional inverse scatterings and non-linear partial differential equations, Proc. Sympos. Pure Math. 43 (1984), 45-70.

[B] G. Borg, Eine Umkerung der Sturm-Liouville Eigenwertarfgabe. Bestimmung def Differentialgleichung durch die Eigenwerte, Acta Math. 78 (1946), 1-96.

[C] A. P. Calderón, On an inverse boundary value problem, Seminar on numerical analysis and its applications to continuum physics, Soc. Brasiliera de Mathematica, Rio de Janeiro (1980), $65-73$. 
[CS] S. Chanillo and E. Sawyer, Unique continuation for $\Delta+v$ and the C. Fefferman-Phong class, Trans. Amer. Math. Soc. (to appear).

[CW] S. Chanillo and R. Wheeden, $L^{p}$ estimates for fractional integrals and Sobolev inequalities with applications to Schrödinger operators, Comm. Partial Differential Equations 10 (1985), 1077-1116.

[F] C. Fefferman, The uncertainty principle, Bull. Amer. Math. Soc. 9 (1983), 129-206.

[GL] I. M. Gelfand and B. M. Levitan, On the determination of a differential equation from its spectral function, Izv. Akad. Nauk. SSSR Ser. Math. 15 (1961), 309-360.

[HN] G. M. Henkin and R. G. Novikov, Uspekhi Mat. Nauk. 42 (1987), 94-153.

[KRS] C. E. Kenig, A. Ruiz and C. Sogge, Sobolev inequalities and unique continuation for second order constant coefficient differential equations, Duke Math. J. 55 (1987), 329-348.

[KV] R. Kohn and M. Vogelius, Determining conductivity by boundary measurements, Comm. Pure Appl. Math. 37 (1984), 289-298.

[L] N. Levinson, The inverse Sturm-Liouville problem, Math. Tidsskr. B. (1949), 25-30.

[NSU] A. Nachman, J. Sylvester and G. Uhlmann, An n-dimensional Borg-Levinson theorem, Comm. Phys. Math. (to appear).

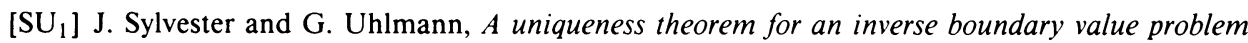
in electrical prospection, Comm. Pure Appl. Math. 39 (1986), 91-112.

$\left[\mathrm{SU}_{2}\right] \ldots$, A global uniqueness theorem for an inverse boundary value problem, Ann. of Math. 125 (1987), 153-169.

Department of Mathematics, Rutgers University, New Brunswick, New Jersey 08903 